\title{
Assessment of the infarct size with ST-elevation from high-resolution standard and orthogonal ECG leads
}

\author{
Mikhail Matveev \\ Institute of Biophysics and Biomedical Engineering, BAS, 1113 Sofia, Bulgaria
}

\begin{abstract}
The high-resolution electrocardiography is known to be a useful tool for extraction and analysis of low-amplitude signal components. We found the high-resolution standard and synthesized orthogonal electrocardiography leads may be useful for precise evaluation of the zone of acute myocardial infarct necrosis. We found high-resolution electrocardiography may be useful for precise location of the site of the myocardial necrosis and assessment of the severity of impaired left-ventricle systolic function of patients with ST-elevation myocardial infarct (STEMI) in the acute phase. High-resolution (1 MHz) ECG from 4 groups were collected: healthy controls (13), patients with anterior (10), inferior STEMI (19) and 12 patients with infero-lateral AMI). The three orthogonal leads X, Y, Z were synthesized from the 12-standard leads by known transformation. The possibility for facilitated and fast performance of this examination in clinical conditions, including emergency, the lack of necessity of specially trained staff for carrying out the examination and interpretation of the results, as well as the very low prime cost, make this electrophysiological method very suitable for application in the routine clinical practice for qualitative and quantitative assessment of patients with acute coronary syndromes.
\end{abstract}

\section{Introduction}

In principle the signal-averaged high-resolution electrocardiography (SAECG) is a technique involving computerized analysis of small segments of a standard electrocardiography (ECG) in order to detect late potentials $[1,2]$. It allows subtraction and analysis of lowamplitude components in the signal, containing important diagnostics information, but inadmissible for analysis using conventional 12-channel ECG. The high resolution SAECG and vectorcardiography (VCG) was employed recently as a method for qualitative and quantitative diagnosis of patients with acute myocardial infarct (AMI). The existing scarce data in world-wide literature about quantitative assessment of patients with AMI by SAECG suggest very high diagnostic value of this method [3-6]. In this research we studied the possibilities to create standards, characterizing ST-elevation myocardial infarct with different location and size using synthesized from SAECG orthogonal X, Y and Z leads and VCG loops.

\section{ECG Data}

High-resolution ECG recordings collected with the developed ECG data acquisition system [1]. The analysis involved 45 ECG recordings collected from 15 patients (average age $70 \pm 12.5$ years) at the intensive coronary unit of the Department of Internal Medicine, Medical University - Sofia, all of them with clinical symptoms, ECG and laboratory signs for AMI with ST-elevation:
- typical angina pain with duration more than $30 \mathrm{~min}$;

- ST-elevation above $1 \mathrm{~mm}$ in two or more neighbouring peripheral leads, or more than 2 $\mathrm{mm}$ in two or more neighbouring precordial leads;

- Serum creatine kinase (CK) activity levels above $180 \mathrm{U} / \mathrm{L}, \mathrm{MB}$-fraction of CK (MB-CK) above $15 \mathrm{U} / \mathrm{L}, \mathrm{MB}-\mathrm{CK}$ above $10 \%$ from the initial $\mathrm{CK}$ value;

- Onset of the clinical symptoms up to 24 hours before admission to hospital.

We involved into analysis recordings from Public Accessible PTB Diagnostic ECG Database [2] - highresolution ECG database (12 standard leads +3 orthogonal leads), because it is collected from healthy volunteers and patients with different heart diseases, which is supported with complete information about the diagnosis, haemodynamics and therapy for each patient.

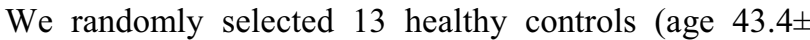
17.8 years) without history, ECG and laboratory indications for cardiovascular or non-cardiac disease, which could influence the ECG potentials. Moreover, we selected 41 patients (age 57.9 12.3 years) with acute AMI (19 patients with inferior AMI, 10 patients with anterior AMI, 12 patients with infero-lateral AMI), which have ECG recordings between 24 to 72 hours from the infarction date.

\section{Methods}

Corresponding author: mgm@biomed.bas.bg 
We study an approach for analysis of high-resolution ECG recordings aiming to provide adequate information about the infarct size and localization. The method is based on assessment of the orthogonal leads $\mathrm{X}, \mathrm{Y}, \mathrm{Z}$ (OECG). Since the developed wireless ECG acquisition system operates only with 12 standard leads, we apply transformations (Eq.1) [3] to derive the synthesized orthogonal Xo,Yo, Zo leads:

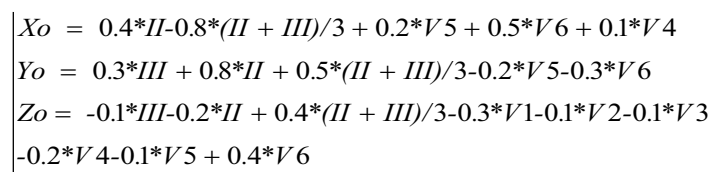

Between lead I and lead II in the standard ECG, there is $60^{\circ}$ zone with projection on the left ventricle, which is not directly reflected by any of the leads [4]. Therefore, to supply additional information, we apply synhresys of the orthogonal X, Y, Z leads, but lead $I I I=2 *(I I+a v R)$ is substituted in transformations (Eq.1) with inversion of the lead $a v R(-a v R)$. In this way, we examined the possibility for improvement of the AMI diagnostics.

The most informative indexes extracted from the orthogonal leads, which show maximal deflection compared to the respective norms, are assessed for each infarct localization type. Taking for a basis an amplitude change of $\Delta A=1 \mathrm{~mm}$ for these indexes, we calculate the equivalent increase in the serum enzyme activity [5]:

$$
\Delta E=\Delta A^{*}(E 2-E 1) /(A 2-A 1)[U / L]
$$

where $E 1$ is the initial enzyme value [U/L], $E 2$ is the peak enzyme activity $[U / L], A 1$ is the initial index amplitude $[\mathrm{mm}], A 2$ corresponds to the index value when maximal amplitude change is reached $[\mathrm{mm}]$. Since patients' admission to hospital after the beginning of the clinical symptoms varies in time and ECG recordings before AMI are usually missing, we substitute $A l$ with the mean value of the respective index for healthy controls. For the same reason (absence of initial 'normal' levels and physiologic variability of $\mathrm{CK}$ and $\mathrm{CK}-\mathrm{MB}$ ), we substitute $E 1$ with the maximal norm level (180 U/L for $\mathrm{CK}, 15 \mathrm{U} / \mathrm{L}$ for $\mathrm{CK}$ $\mathrm{MB})$.

\section{Results}

The practical implementation of the high-resolution ECG acquisition system is demonstrated in Fig.1. Here is presented an example of ECG recordings (12 standard leads + synthesized orthogonal leads ) from a patient with acute anterior AMI, which is admitted within $24 \mathrm{~h}$ from the beginning of the clinical symptoms. Taking into account that the high-resolution ECG recordings of the healthy controls do not contain any of the components, which are characteristic for the acute or undergone myocardionecrosis, we estimated the mean values of the indexes, which are known to show changes during lesion and myocardial necrosis [6, 7]. The indexes are measured from: (i) Frank orthogonal leads (X,Y,Z); (ii) the synthesized orthogonal leads (X1,Y1,Z1); (iii) the synthesized orthogonal leads with inversion of the lead $a v R(\mathrm{X} 2, \mathrm{Y} 2, \mathrm{Z} 2)$. The results are summarized in Table 1. to Table 4 .
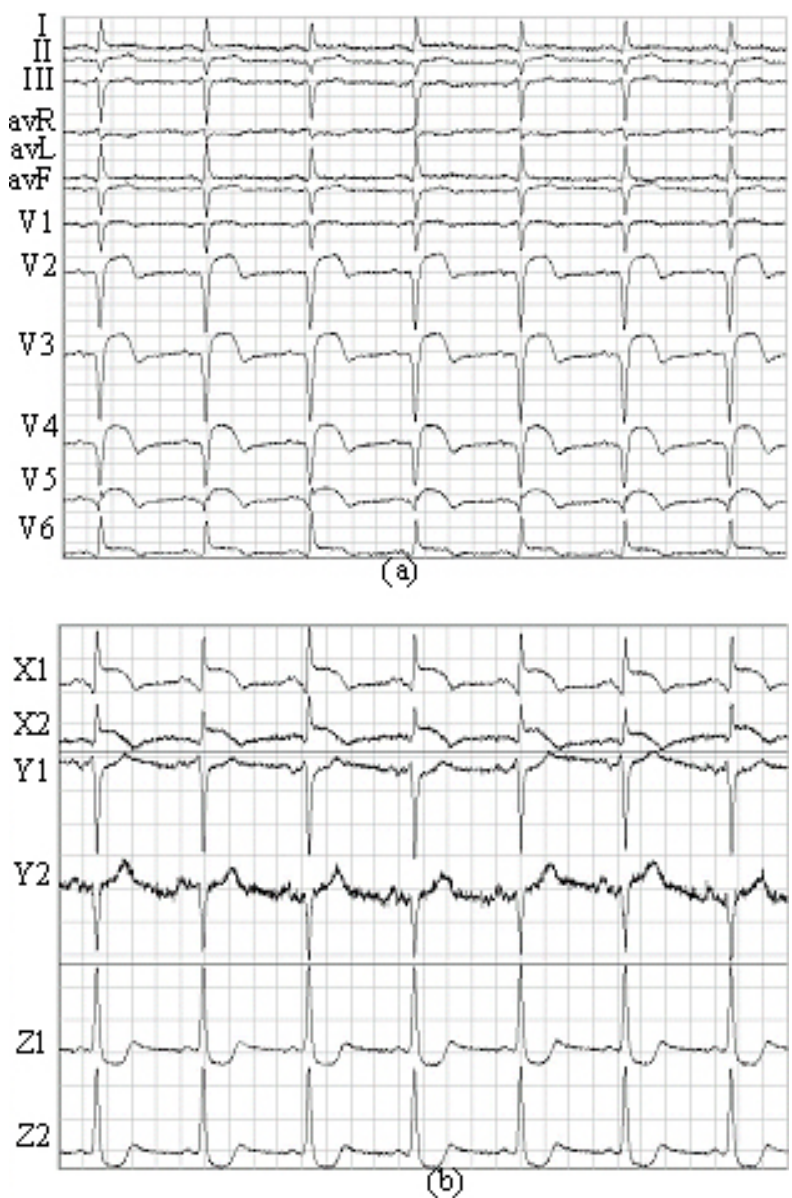

Figure 1. Example of high-resolution ECG recordings: (a) 12 standard leads; (b) synthesized orthogonal leads

Table 1. Indexes for healty controls $(\mathrm{N}=13)$.

\begin{tabular}{|cccc|}
\hline $\begin{array}{c}\text { OECG } \\
\text { Leads }\end{array}$ & $\begin{array}{c}\text { Q-wave } \\
\text { amplitude } \\
\text { [mm] }\end{array}$ & $\begin{array}{c}\text { R-wave } \\
\text { amplitude } \\
\text { [mm] }\end{array}$ & $\begin{array}{c}\text { ST-segme nf } \\
\text { Elevation } \\
\text { [mm] }\end{array}$ \\
\hline $\mathbf{X}$ & $1,6 \pm 1,4$ & $15,7 \pm 6,8$ & - \\
$\mathbf{X} 1$ & $0,8 \pm 0,3$ & $14,6 \pm 6,5$ & - \\
$\mathbf{X} 2$ & $0,7 \pm 06$ & $4,95 \pm 4,0$ & - \\
$\mathbf{Y}$ & $0,9 \pm 0$, & $10,3 \pm 5,7$ & - \\
$\mathbf{Y} 1$ & $1,4 \pm 0,6$ & $10,0 \pm 6,6$ & - \\
$\mathbf{Y 2}$ & $1,6 \pm 1,1$ & $24,9 \pm 11,98$ & $0,85 \pm 0,2$ \\
$\mathbf{Z}$ & $3,6 \pm 2,1$ & $7,3 \pm 2,9$ & - \\
$\mathbf{Z 1}$ & $2,7 \pm 1,98$ & $7,3 \pm 2,5$ & - \\
$\mathbf{Z 2}$ & $2,5 \pm 1,3$ & $7,95 \pm 2,5$ & - \\
& & & \\
\hline
\end{tabular}


Table 2. Indexes for patients with inferior AMI $(\mathrm{N}=19)$.

\begin{tabular}{|cccc|}
\hline $\begin{array}{c}\text { OECG } \\
\text { Leads }\end{array}$ & $\begin{array}{c}\text { Q-wave } \\
\text { amplitude } \\
\text { [mm] }\end{array}$ & $\begin{array}{c}\text { R-wave } \\
\text { amplitude } \\
\text { [mm] }\end{array}$ & $\begin{array}{c}\text { ST-segment } \\
\text { Elevation } \\
\text { [mm] }\end{array}$ \\
\hline X & $2(\mathrm{n}=1)$ & $12,6 \pm 4,33$ & $2(\mathrm{n}=1)$ \\
$\mathbf{X 1}$ & $1.5(\mathrm{n}=1)$ & $12,11 \pm 2.2$ & $2(\mathrm{n}=1)$ \\
$\mathbf{X 2}$ & $1,25 \pm 0,35$ & $? 74 \pm 3,31$ & $1,5(\mathrm{n}=1)$ \\
$\mathbf{Y}$ & $2,43 \pm 1,66$ & $6,-5 \pm 2,99$ & $0,86 \pm 0,84$ \\
Y1 & $4,17 \pm 2,40$ & $5,75 \pm 2,85$ & $0,92 \pm 0,99$ \\
Y2 & $4,38 \pm 2,55$ & $18,87 \pm 7,1$ & $1,26 \pm 1,39$ \\
Z & $5,32 \pm 3,39$ & $6,83 \pm 3,69$ & $0,13 \pm 0,28$ \\
Z1 & $5,11 \pm 3,87$ & $8,36 \pm 4,47$ & $0,21 \pm 0,38$ \\
Z2 & $4,48 \pm 3,64$ & $8,5 \pm 4,57$ & $0,21+0,38$ \\
\hline
\end{tabular}

Table 3. Indexes for patients with anterior AMI $(\mathrm{N}=10)$.

\begin{tabular}{|cccc|}
\hline $\begin{array}{c}\text { OECG } \\
\text { Leads }\end{array}$ & $\begin{array}{c}\text { Q-wave } \\
\text { amplitude } \\
\text { [mm] }\end{array}$ & $\begin{array}{c}\text { R-wave } \\
\text { amplitude } \\
\text { [mm] }\end{array}$ & $\begin{array}{c}\text { ST-segment } \\
\text { Elevation } \\
{[\mathrm{mm}]}\end{array}$ \\
\hline $\mathbf{X}$ & $0,75 \pm 1,06$ & $10,75 \pm 2,18$ & $0,37 \pm 0,69$ \\
$\mathbf{X 1}$ & $0,75 \pm 1,06$ & $10,0 \pm 2,07$ & $0,37 \pm 0,69$ \\
$\mathbf{X 2}$ & $1,2, \pm 2,15$ & $1,0 \pm 1,30$ & $0,06 \pm 0,17$ \\
$\mathbf{Y}$ & $1,37 \pm 1,66$ & $9,56 \pm 4,51$ & $0,68 \pm 1,27$ \\
Y1 & $1,44 \pm 2,49$ & $7,68 \pm 5,47$ & $1,0 \pm 1,38$ \\
Y2 & $2,37 \pm 2,66$ & $25,62 \pm 10,5$ & $1,25 \pm 1,77$ \\
Z & $1,43 \pm 1,29$ & $7,81 \pm 4,17$ & 0 \\
Z1 & $1,06 \pm 0,94$ & $11,12 \pm 2,23$ & 0 \\
Z2 & $1,06 \pm 0,94$ & $11,81 \pm 2,17$ & 0 \\
\hline
\end{tabular}

Table 4. Indexes for patients with inf.-lat. AMI $(\mathrm{N}=10)$.

\begin{tabular}{|cccc|}
\hline $\begin{array}{c}\text { OECG } \\
\text { Leads }\end{array}$ & $\begin{array}{c}\text { Q-wave } \\
\text { amplitude } \\
\text { [mm] }\end{array}$ & $\begin{array}{c}\text { K-wave } \\
\text { amplitude } \\
\text { [mm] }\end{array}$ & $\begin{array}{c}\text { ST-segment } \\
\text { Elevation } \\
\text { [mm] }\end{array}$ \\
\hline $\mathbf{X}$ & $1,3 \pm 1,25$ & $15,5 \pm 3,83$ & $0,35 \pm 0,57$ \\
$\mathbf{X 1}$ & $1,1 \pm 0,99$ & $15,4 \pm 3,88$ & $0,35 \pm 0,57$ \\
$\mathbf{X 2}$ & $0,55 \pm 0,95$ & $-, j \pm 6,0$ & $0,15 \pm 0,33$ \\
$\mathbf{Y}$ & $3,6 \pm 2,13$ & $5,6 \pm 2,35$ & $0,4 \pm 0,45$ \\
Y1 & $6,35 \pm 2,59$ & $0,95 \pm 1,70$ & $0,5 \pm 0,57$ \\
Y2 & $6,25 \pm 4,03$ & $15,05 \pm 8,8$ & $1,15 \pm 0,91$ \\
Z & $3,95 \pm 2,56$ & $5,25 \pm 2,53$ & $0,1 \pm 0,31$ \\
Z1 & $4,2 \pm 2,23$ & $5,85 \pm 3,30$ & $0,05 \pm 0,15$ \\
Z2 & $4,05 \pm 2,20$ & $6,25 \pm 3,33$ & 0 \\
\hline
\end{tabular}

We determined the indexes, which show the most significant dynamics for the respective infarct localization compared to the norm of the healthy controls (Table 1):

- inferior AMI: Q-wave amplitude in Z1, Z2, Y1, Y2; Rwave amplitude in Y1 и Y2;

- anterior AMI: Q-wave amplitude in X2, Y2; R-wave amplitude in $\mathrm{X}, \mathrm{X} 1, \mathrm{X} 2, \mathrm{Y} 1$;

- infero-lateral AMI: Q-wave amplitude in Y1,Y2, Z1, Z2; R-wave amplitude in Y1,Y2;

Table 5 contains the equivalent changes of the calculated serum activity (CK and CK-MB) for Q-wave and R-wave amplitude change of $1 \mathrm{~mm}$. The correlation between the electrophysiological and enzyme variables gives the possibility for estimation of the volume of the necrotic tissue in gram/equivalents, after preliminary calculation on the basis of serum peak levels of the enzyme activity, taken from $[4,6]$.
Table 5. Enzyme activity increase for $1 \mathrm{~mm}$ Q-wave and R-wave amplitude change.

\begin{tabular}{|c|c|c|}
\hline \multicolumn{3}{|c|}{ Infer ior AMII } \\
\hline$\underset{\mathrm{DEX}}{\mathrm{IN}}$ & $\begin{array}{c}\mathrm{CK} \\
{[\mathrm{U} / \mathrm{L}]}\end{array}$ & $\begin{array}{c}\text { CK-MB } \\
{[\mathrm{U} / \mathrm{L}]}\end{array}$ \\
\hline $\mathrm{Zl}_{0}$ & $348,6 \pm 168,5$ & $26,8 \pm 3,7$ \\
\hline $\mathrm{Z} 2 \mathrm{O}$ & $249,8 \pm 28,8$ & $28,1 \pm 4,3$ \\
\hline $\mathrm{Y}_{0}$ & $433,2 \pm 48,9$ & $14,8 \pm 2,7$ \\
\hline $\mathrm{Y} 2_{\mathrm{O}}$ & $668,1 \pm 283,4$ & $13,9 \pm 4,2$ \\
\hline $\mathrm{Yl}_{\mathrm{R}}$ & $175,5 \pm 76,2$ & $18,7 \pm 2,1$ \\
\hline $\mathrm{Y} 2_{\mathrm{R}}$ & $76,9=13,9$ & $18,4 \pm 1,6$ \\
\hline \multicolumn{3}{|c|}{ Infero-later al AMII } \\
\hline$\underset{\mathrm{DEX}}{\mathrm{IN}}$ & $\begin{array}{c}\mathrm{CK} \\
{[\mathrm{U} / \mathrm{L}]}\end{array}$ & $\begin{array}{c}\text { CK-MB } \\
{[\mathrm{U} / \mathrm{L}]}\end{array}$ \\
\hline $\mathrm{Yl}_{\mathrm{Q}}$ & $166,55 \pm 88,9$ & $14,1 \pm 3,9$ \\
\hline $\mathrm{Y} 2 \mathrm{O}$ & $201,1 \pm 97,7$ & $14,9 \pm 2,9$ \\
\hline $\mathrm{Zl}_{\mathrm{Q}}$ & $263,0 \pm 288,4$ & $28,1 \pm 4,9$ \\
\hline $22_{0}$ & $154,6 \pm 66,2$ & $28,5 \pm 4,7$ \\
\hline $\mathrm{Yl}_{\mathrm{R}}$ & $179,8 \pm 101,8$ & $19,0 \pm 1,4$ \\
\hline $\mathrm{Y} 2 \mathrm{R}$ & $103,8 \pm 64,8$ & $19,5 \pm 0,8$ \\
\hline \multicolumn{3}{|c|}{ Anterior AMII } \\
\hline$\underset{\text { DEX }}{\text { IN }}$ & $\begin{array}{c}\text { CK } \\
{[\mathrm{U} / \mathrm{L}]}\end{array}$ & $\begin{array}{c}\text { CK-MB } \\
{[\mathrm{U} / \mathrm{L}]}\end{array}$ \\
\hline $\mathrm{X} 2 \mathrm{O}$ & $266,0 \pm 193,7$ & $15,6 \pm 6,7$ \\
\hline $\mathrm{Y} 2 \mathrm{O}$ & $647,5 \pm 427,7$ & $14,3 \pm 4,9$ \\
\hline $\mathrm{X} 1_{R}$ & $159,6 \pm 63,7$ & $5,48 \pm 2,3$ \\
\hline $\mathrm{X} 2_{\mathrm{R}}$ & $115,8 \pm 47,1$ & $6,88 \pm 3,06$ \\
\hline $\mathrm{Yl}_{\mathrm{R}}$ & $168,8 \pm 3,7$ & $3,1=0,88$ \\
\hline
\end{tabular}

\section{References}

1. PTB Diagnostic ECG Database. URL: http://physionet.org/physiobank/database/ptbdb/.

2. Levkov $\mathrm{Ch}$. Orthogonal electrocardiogram derived from the limb and chest electrodes of the conventional 12-lead system, Medical \& Biol. Engineering \& Computing, 25:155-164 (1987).

3. Menown A., Adgey A. Improving the ECG classification of inferior and lateral myocardial infarction by inversion of lead aVR, Heart, 83:657660 (2000).

4. Hamidi E., V1. Ivanov, M. Matveev. Size of the necrotic zone after acute myocardial infarct, estimated by electrocardiographic parameters and serial activity of serum enzymes for myocardial necrosis, Modern Medicine, 11:39-44 (1987) (in bulgarian).

5. Birnbaum Y., G. Wagner. The initial electrocardiographic pattern in acute myocardial infarction: Correlation with infarct size, Journal of Electrocardiology, 32:122-127 (1999).

6. Grǿttum P., J. Kjekshus. A comparison of cumulated CK Release with three vectorcardiographic methods of estimating myocardial infarct size, Journal of Electrocardiology, 19:337-346 (1986).

7. Matveev M., V. Krasteva, St. Naydenov, T. Donova. Possibilities of signal-averaged orthogonal and vector electrocardiography for locating and size evaluation of acute myocardial infarction with ST-elevation, Anatol J Cardiol. Suppl.1:193-197 (2007). 